\title{
Index of Volume 192 (1990)
}

\section{Number 1/2}

F. C. Hawthorne

Structural hierarchy in $\mathrm{M}^{[6]} \mathrm{T}^{[4]} \phi_{n}$ minerals . . . . . . . . . . . . 1

S. Haussühl

Elastic properties of cubic $\mathrm{X}\left(\mathrm{BrO}_{3}\right)_{2} \cdot 6 \mathrm{H}_{2} \mathrm{O}, \mathrm{X}=\mathrm{Mg}, \mathrm{Co}, \mathrm{Ni}, \mathrm{Cu}, \mathrm{Zn}$

M. Vlassi, G. Germain, K. Barlos, P. Mamos, L. S. Refaat

Crystal structure of $N^{x}$-tritylleucyl-(1-benzotriazolyl ester) . . . . .

J. Podlahová, J. Podlaha, I. Císařová

Crystal structure of calcium diphenylphosphineacetate . . . . . . . .

C. Chakrabarti, A. Podder, J. K. Dattagupta

Crystal structure of 2-hydroxy-4-methylpyrimidine hydrochloride $\mathrm{C}_{5} \mathrm{H}_{6} \mathrm{~N}_{2} \mathrm{O} \cdot \mathrm{HC}$

A. G. Nord, T. Ericsson, P.-E. Werner

The crystal structure of iron(II) tetrametaphosphate, $\mathrm{Fe}_{2} \mathrm{P}_{4} \mathrm{O}_{12}$. . . .

L. Bohatý, J. Liebertz

Electro-optic and electrostrictive properties of the low-temperature phase of barium borate $\mathrm{BaB}_{2} \mathrm{O}_{4}$.

H. Küppers

The structure of a second modification of cobalt(II) dihydrogen diphthalate hexahydrate

E. R. T. Tiekink, M. S. Hundal, G. Sood, P. Kapoor, N. S. Poonia

Crystal and molecular structure of sodium dihydrogen tri(3,5-dinitrobenzoate)

M. Sacerdoti, V. Bertolasi, V. Ferretti, C. A. Accorsi

A redetermination of the crystal structure of barium hydroxide octahydrate $\mathrm{Ba}(\mathrm{OH})_{2} \cdot 8 \mathrm{H}_{2} \mathrm{O}$

H. Sov'a, J. Macavei, H. Schulz

The crystal structure of berlinite $\mathrm{AlPO}_{4}$ at high pressure .

S. Haussühl

Elastic and thermoelastic properties of isotypic $\mathrm{KClO}_{4}, \mathrm{RbClO}_{4}, \mathrm{CsClO}_{4}$, $\mathrm{TlClO}_{4}, \mathrm{NH}_{4} \mathrm{ClO}_{4}, \mathrm{TlBF}_{4}, \mathrm{NH}_{4} \mathrm{BF}_{4}$ and $\mathrm{BaSO}_{4}$ 
Short Communications

J. D. Tornero, J. Fayos

Single crystal structure refinement of $\mathrm{MnCl}_{2}$

K. Schubert

On the polytypism of $\mathrm{SiC}$

Book Revieus

P. J. Goodhaw, J. Humphreys

Electron Microscopy and Analysis (2. Auflage). (Rez.: G. Wagner) . . 1:53

B. K. Vaynshteyn, A. A. Chernov (Eds.).

Modern Crystallography. (Rez.: J. Bohm).

R. Rykart

Quarz-Monographie. Die Eigenheiten von Bergkristall, Rauchquarz, Amethyst und anderen Varietäten. (Rez.: H.-J. Höbler)

E. B. Vinberg

Linear Representations of Groups. (Rez.: K. Schmüdgen)

I. B. Bersuker, V. Z. Polinger

Vibronic Interactions in Molecules and Crystals. (Rez.: A. Kühnel) .

H.-G. Bigalke

Heinrich Heesch, Kristallgeometrie. Parkettierungen, Vierfarbenforschung. (Rez.: P. Paufler)

K. H. Kuo (Ed.)

The Application of Electron Microscopy to Materials Science Proceedings of an International Workshop, held in Gauonzhou, China, August 1988. (Rez.: W. Neumann)

P. P. Pashinin (Ed.)

Laser-induced Raman spectroscopy in crystals and gases. Proceedings of

the Institute of General Physics. (Rez.: W. E. Steger)

\section{Number $3 / 4$}

K. Schubert

On some homeotypes of $\mathrm{Si}$

P. Benna, M. Tribaudino, G. Zanini, E. Bruno

The crystal structure of $\mathrm{Ca}_{0.8} \mathrm{Mg}_{1.2} \mathrm{Si}_{2} \mathrm{O}_{6}$ clinopyroxene $\left(\mathrm{Di}_{80} \mathrm{En}_{20}\right)$ at $T=-130^{\circ}, 25^{\circ}, 400^{\circ}$ and $700^{\circ} \mathrm{C}$

B. Solcher, G. Klöss, P. Paufler

Slip band formation during bending of GaAs wafers. 
E. Hellner, W. B. Pearson

Laves-phase arrangements of metal atoms in some oxides . . . . . . 219

M. Ledésert, A. Rebbah, Ph. Labbé

$\mathrm{Hg}_{9} \mathrm{P}_{5} \mathrm{I}_{6}$ : a new mercury(I, II) structural determination

E. Lissel, M. Jansen, E. Jansen, G. Will

Bestimmung der Kristallstruktur von $\mathrm{T}-\mathrm{Na}_{3} \mathrm{PO}_{4}$ mit Röntgen- und Neutronenpulvertechniken

S. Haussühl

Optical activity in centrosymmetric crystals?

M. L. Fornasini, A. Palenzona

The crystal structure of $\mathrm{Er}_{3} \mathrm{Ru}_{2}$

N. Galešić, A. Vlahov

Crystal structure of 5-(p-bromophenyl)-N,N-bis(2-chloroethyl)-2-furamide

\section{New Crystal Structures}

M. Somer, M. Hartweg, K. Peters, H. G. von Schnering

Crystal structure of tetrapotassium diphosphidoberyllate, $\mathrm{K}_{4} \mathrm{BeP}_{2}$. . 263

M. Somer, M. Hartweg, K. Peters, H. G. von Schnering

Crystal structure of tetrapotassium diarsenidoberyllate, $\mathrm{K}_{\mathbf{4}} \mathrm{BeAs}_{2}$. . .

M. Somer, K. Peters, H. G. von Schnering

Crystal structure of dipotassium sodium diphosphidogallate, $\mathrm{K}_{2} \mathrm{NaGaP}_{2}$

M. Somer, D. Thiery, M. Hartweg, L. Walz, T. Popp, K. Peters, H. G. von Schnering

Crystal structure of tricaesium diarsenidoaluminate, $\mathrm{Cs}_{3} \mathrm{AlAs}_{2}$. . . . 269

M. Somer, K. Peters, D. Thiery, H. G. von Schnering

Crystal structure of trirubidium diphosphidogallate, $\mathrm{Rb}_{3} \mathrm{GaP}_{2}$

M. Somer, K. Peters, T. Popp, H. G. von Schnering

Crystal structure of tricaesium diarsenidogallate, $\mathrm{Cs}_{3} \mathrm{GaAs}_{2}$. . . .

C. Sudarsanakumar, M. Rajasekhar Rao, S. Srinivasan, B. Chandrasekhar, S. R. Ramadas

Crystal structure of 2-methoxy-7,7-dimethyl-N-p-tolyl-pyrrolono\{f,g $\}$ $7 \mathrm{H}$-xanthen, $\mathrm{C}_{24} \mathrm{H}_{21} \mathrm{NO}_{3}$

U. Flörke, H.-J. Haupt

Crystal structure of $\mu$-hydrido-tri- $\mu$-diphenylphosphido-di- $\mu_{4}$-phenylphosphido-octacarbonyl-tetraangulo-tetrarhenium dichloromethane, $\mathrm{Re}_{4}(\mathrm{H})\left(\mathrm{P}\left(\mathrm{C}_{6} \mathrm{H}_{5}\right)_{2}\right)_{3}\left(\mathrm{P}\left(\mathrm{C}_{6} \mathrm{H}_{5}\right)\right)_{2}(\mathrm{CO})_{8} \mathrm{CH}_{2} \mathrm{Cl}_{2}$. . . . . . . . . .

U. Flörke, H.-J. Haupt

Crystal structure of $\mu$-iodo- $\mu$-oxo-tetra- $\mu$-diphenylphosphido- $\mu_{4}$-phenylphosphido-octacarbonyl-tetraangulo-tetrarhenium,

$\mathrm{Re}_{4}(\mathrm{I})(\mathrm{O})\left(\mathrm{P}\left(\mathrm{C}_{6} \mathrm{H}_{5}\right)_{2}\right)_{4}\left(\mathrm{P}\left(\mathrm{C}_{6} \mathrm{H}_{5}\right)\right)(\mathrm{CO})_{8} \ldots$ 
U. Flörke, H.-J. Haupt

Crystal structure of $\mu$-iodo- $\mu$-oxo- $\mu$-hydrido-tri- $\mu$-diphenylphosphido$\mu_{4}$-phenylphosphido-octacarbonyl-tetraangulo-tetrarhenium trichloro-

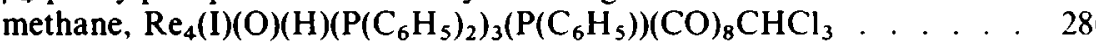

U. Flörke, H.-J. Haupt

Crystal structure of cis-iodo-diphenylphosphine-tetracarbonyl-rhenium. $\operatorname{IRe}(\mathrm{CO})_{4}\left(\mathrm{P}_{\left.\left(\mathrm{C}_{6} \mathrm{H}_{5}\right)_{2} \mathrm{H}\right) \ldots \ldots} \ldots \ldots\right.$

U. Flörke, H.-J. Haupt

Crystal structure of di- $\mu$-iodo- $\mu$-tetraphenyl-diphosphanoxide-bis(tricarbonyl-rhenium), $\operatorname{Re}_{2}(\mathrm{I})_{2}\left(\mathrm{P}\left(\mathrm{C}_{6} \mathrm{H}_{5}\right)_{2} \mathrm{OP}\left(\mathrm{C}_{6} \mathrm{H}_{5}\right)_{2}\right)(\mathrm{CO})_{6} \ldots \ldots$. . . .

U. Flörke, H.-J. Haupt

Crystal structure of $\mu_{3}$-hydrido- $\mu_{3}$-iodo-tri- $\mu$-diphenylphosphido-hexacarbonyl-triangulo-trirhenium, $\operatorname{Re}_{3}(\mathrm{H})(\mathrm{I})\left(\mathrm{P}\left(\mathrm{C}_{6} \mathrm{H}_{5}\right)_{2}\right)_{3}(\mathrm{CO})_{6} \ldots \ldots$

U. Flörke, H.-J. Haupt

Crystal structure of $\mu_{3}$-hydrido- $\mu_{3}$-bromo-tri- $\mu$-diphenylphosphido-hexacarbonyl-triangulo-trirhenium, $\operatorname{Re}_{3}(\mathrm{H})(\mathrm{Br})\left(\mathrm{P}\left(\mathrm{C}_{6} \mathrm{H}_{5}\right)_{2}\right)_{3}(\mathrm{CO})_{6}$

U. Flörke, H.-J. Haupt

Crystal structure of $\mu$-iodo- $\mu$-hydrido-tetra- $\mu$-diphenylphosphido- $\mu_{4^{-}}$ phenylphosphido-octacarbonyl-tetraangulo-tetrarhenium trichloromethane dichloromethane, $\mathrm{Re}_{4}(\mathrm{I})(\mathrm{H})\left(\mathrm{P}\left(\mathrm{C}_{6} \mathrm{H}_{5}\right)_{2}\right)_{4}\left(\mathrm{P}\left(\mathrm{C}_{6} \mathrm{H}_{5}\right)\right)(\mathrm{CO})_{8^{-}}$ $\left(\mathrm{CHCl}_{3}\right)_{0.87}\left(\mathrm{CH}_{2} \mathrm{Cl}_{2}\right)_{0.77}$

L. Walz

Crystal structure of 4'-(4-propylphenyl)phenyl-4"-butylbenzoate, $\left(\mathrm{C}_{4} \mathrm{H}_{9}\right)\left(\mathrm{C}_{6} \mathrm{H}_{4}\right) \mathrm{COO}\left(\mathrm{C}_{6} \mathrm{H}_{4}\right)\left(\mathrm{C}_{6} \mathrm{H}_{4}\right)\left(\mathrm{C}_{3} \mathrm{H}_{7}\right) \ldots \ldots . . . . . .308$

$F$. Knoch, $H$. Wiedenfeld

Crystal structure of $11 \beta, 17$-dihydroxy-21-ureido-pregn-4-en-3.20-dion. $\mathrm{C}_{22} \mathrm{H}_{32} \mathrm{~N}_{2} \mathrm{O}_{5}$ 